\title{
Atividade anti-helmíntica dos extratos aquoso e etanólico do fruto da Morinda citrifolia sobre Ascaridia galli
}

\author{
Anthelmintic activity of aqueous and ethanolic extracts of Morinda citrifolia fruit on Ascaridia galli \\ Danilo R. Barros Brito ${ }^{1}$; Rozeverter Moreno Fernandes ${ }^{2 *}$; Maria Zenaide de Lima C. M. Fernandes ${ }^{3}$; \\ Marcos Daniel de S. Ferreira ${ }^{4}$; Fernanda R. L. Rolim'; Manoel L. da Silva Filho ${ }^{4}$ \\ ${ }^{1}$ Curso de Pós-graduação em Ciência Animal, Universidade Federal do Piauí - UFPI \\ ${ }^{2}$ Departamento de Morfofisiologia Veterinária, Centro de Ciências Agrárias, Universidade Federal do Piauí - UFPI \\ ${ }^{3}$ Departamento das Clínicas, Universidade Estadual do Piaiuí - UESPI \\ ${ }^{4}$ Curso de Medicina Veterinária, Universidade Federal do Piauí - UFPI. \\ ${ }^{5}$ Universidade Federal do Piauí - UFPI. \\ Recebido em 14 de Abril de 2009 \\ Aceito em 1 de Junho de 2009
}

\section{Resumo}

A atividade anti-helmíntica dos extratos aquoso e etanólico do fruto da Morinda citrifolia (noni) foi avaliada em aves poedeiras naturalmente infectadas por Ascaridia galli. A atividade anti-helmíntica in vitro foi determinada em parasitos adultos. O extrato aquoso e etanólico foram testados nas seguintes concentraçóes: 1,69; 3,37; 6,74; 13,48 e 26,96 mg.mL $\mathrm{mL}^{-1}$ e 4,17; 8,34; 16,68; 33,36 e 66,72 mg. $\mathrm{mL}^{-1}$, respectivamente. A atividade anti-helmíntica in vivo foi determinada administrando-se, durante três dias consecutivos, o extrato aquoso $\left(50,1 \mathrm{mg} \cdot \mathrm{mL}^{-1}\right)$ e etanólico $\left(24,6 \mathrm{mg} \cdot \mathrm{mL}^{-1}\right)$, sendo $10 \mathrm{~mL} \cdot \mathrm{kg}^{-1}$. Posteriormente, as aves foram sacrificadas e necropsiadas para contagem dos helmintos remanescentes. Os dados obtidos foram analisados estatisticamente, utilizando-se o teste de Student-Newman-Keuls. Nas concentraçôes 13,48 e 26,96 mg. $\mathrm{mL}^{-1}$, o extrato aquoso apresentou taxa de mortalidade de 46,67 e 50\%, respectivamente, sendo estatisticamente diferente do controle negativo $(\mathrm{P}<0,05)$. O extrato etanólico apresentou diferença significativa do controle negativo (diluente) $(\mathrm{P}<0,05)$ para as concentraçôes 33,36 e $66,72 \mathrm{mg} \cdot \mathrm{mL}^{-1}$, expressando taxa de mortalidade de 66,67 e 76,67\%, respectivamente. No teste in vivo, o extrato aquoso do fruto do noni apresentou percentual de eliminaçâo de $27,08 \%$, diferindo estatisticamente do grupo controle. Não houve diferença significativa entre os tratamentos com extrato etanólico e controle $(\mathrm{P}>0,05)$. Conclui-se que a atividade anti-helmíntica do fruto do noni, no teste in vitro, apresentou resultados satisfatórios, havendo necessidade de estudos com maiores concentraçóes no teste in vivo.

Palavras-chave: Aves, Ascaridia galli, fitoterapia, Morinda citrifolia, atividade anti-helmíntica.

\begin{abstract}
The anthelmintic activity of aqueous and ethanolic extracts of Morinda citrifolia fruit (noni) was evaluated in chicken naturally infected by Ascaridia galli. The anthelmintic activity in vitro was determined in adult parasites. The aqueous and ethanolic extracts were used in the following concentrations: 1.69; 3.37; 6.74; 13.48 e $26.96 \mathrm{mg} \cdot \mathrm{mL}^{-1}$ and 4.17; 8.34; $16.68 ; 33.36$ and $66.72 \mathrm{mg} \cdot \mathrm{mL}^{-1}$, respectively. The anthelmintic activity in vivo was determined by the administration of $10 \mathrm{~mL} \cdot \mathrm{kg}^{-1}$ of the aqueous $\left(50.1 \mathrm{mg} \cdot \mathrm{mL}^{-1}\right)$ and ethanolic $\left(24.6 \mathrm{mg} \cdot \mathrm{mL}^{-1}\right)$ extracts during three consecutive days. Later the chickens were euthanized and necropsy was performed in order to count the remaining helminths. The data were analyzed by the Student-Newman-Keuls test. In the concentrations of 13.48 and $26.96 \mathrm{mg} \cdot \mathrm{mL}^{-1}$, the aqueous extract demonstrated mortality of 46.67 and $50 \%$, respectively, there was a significative difference from the negative control $(\mathrm{P}<0.05)$. The ethanolic extract presented statistical difference from the negative control (diluent) $(\mathrm{P}<0.05)$ for the concentrations of 33.36 and $66.72 \mathrm{mg} \cdot \mathrm{mL}^{-1}$, expressed by a mortality rate of 66.67 and $76.67 \%$, respectively. In the in vivo test, the aqueous extract of noni fruit showed $27.08 \%$ of elimination, deferring statistically from the control group. There was no statistical difference between the ethanolic extract treatments and the control $(P>0.05)$. It follows that the anthelmintic activity of noni fruit test showed satisfactory results in vitro, there is a need for studies in higher concentrations in the in vivo test.
\end{abstract}

Keywords: Birds, Ascaridia galli, phytotherapy, Morinda citrifolia, anthelmintic activity.

\footnotetext{
*Autor para correspondência: Rozeverter Moreno Fernandes

Departamento de Morfofisiologia Veterinária, Centro de Ciências Agrárias,

Universidade Federal do Piauí - UFPI, Campus Agrários da Socopo - CCA,

s/n, CEP 64049-550, Teresina - PI, Brasil;

e-mail: zmoreno@ufpi.br; Apoio: UFPI, CNPq e FAPEMA
} 


\section{Introdução}

Dados da Organização Mundial de Saúde (OMS) indicaram que cerca de $80 \%$ da população mundial já usou algum tipo de planta na busca de alívio de alguma sintomatologia dolorosa ou desagradável. Desse total, a indicação médica foi de, pelo menos, 30\% (MARTINS et al., 2000).

As plantas medicinais são importantes por fornecerem matéria-prima para a síntese de drogas, além de serem utilizadas como agentes terapêuticos. $\mathrm{O}$ emprego das plantas é supervalorizado no uso tradicional com base nos seus benefícios medicinais. Dessa forma, torna-se imprescindível o conhecimento sobre a dose e a parte empregada da planta, além de suas propriedades terapêuticas, pois existem aquelas que são altamente tóxicas, mesmo em pequenas doses (ZHAN; ZHOU, 2003).

A validação científica dos fitoterápicos é uma etapa inicial obrigatória para a utilização correta de plantas medicinais ou de seus compostos ativos. A total aceitação de drogas derivadas de plantas e a fitoterapia da medicina científica só poderấo ocorrer se esses produtos cumprirem os mesmos critérios de eficácia, segurança e controle de qualidade que os produtos sintéticos (RATES, 2001). Os produtos derivados de plantas devem ter eficácia avaliada e confirmada, assim como deve ser garantida que sua administração a organismos vivos ocorra sem riscos para a saúde (CAMURÇA-VASCONCELOS et al., 2005). Assim, a fitoterapia pode contribuir para aumentar a produtividade, uma vez que reduz o uso de anti-helmínticos convencionais, além de estender a vida útil dos produtos químicos disponíveis (VIEIRA et al., 1999).

Diversas plantas são citadas popularmente como tendo atividade anti-helmíntica. Dentre elas, tem-se Morinda citrifolia, conhecida por noni. É uma pequena árvore da família das Rubiaceae, originária do Sudoeste da Ásia, tendo sido difundida pelo homem através da Índia e do Oceano Pacífico até as ilhas da Polinésia Francesa. O emprego tradicional do noni pelos polinésios é atribuído aos efeitos relacionados com atividade antibacteriana, antiviral, antifúngica, antitumoral, anti-helmíntica, analgésica, anti-inflamatória, hipotensora e imunoestimulante, usado há mais de 2000 anos (WANG et al., 2002).

A ascaridíase é uma parasitose causada por Ascaridia galli, sendo comum na avicultura doméstica. As aves novas são mais susceptíveis do que as adultas, e os vermes adultos podem causar obstrução intestinal e morte das aves (FREITAS, 1977). Em estudo realizado na Dinamarca, a prevalência de $A$. galli foi de $100 \%$ em frangos criados no sistema Colonial/Caipira e orgânico, enquanto para o sistema industrial foi de $25 \%$. A alta prevalência de $A$. galli e outros helmintos nesse tipo de produção, provavelmente, contribuem para a mortalidade das aves (PERMIN et al., 2002).

Dentro da perspectiva da produção de frangos e ovos, a utilizaçáo de sistemas orgânicos e outros não intensivos, vêm crescendo em importância em vários países em contraste com o sistema convencional. Essas criaçóes são afetadas por várias mudanças no manejo, tais como: acesso a áreas de pasto e proibição no uso de medicamentos preventivos, incluindo anti-parasitários. Tal modelo implica o aperfeiçoamento do manejo e alimentaçáo das aves, uma vez que, tendo acesso a piquetes, as aves teráo contato com ovos, larvas e hospedeiros intermediários de diversos helmintos (THAMSBORG et al., 1999). Diante disso, o objetivo deste trabalho foi avaliar a atividade anti-helmíntica do fruto de M. citrifolia sobre A. galli.

\section{Material e Métodos}

\section{Matéria vegetal}

Os frutos da planta $M$. citrifolia foram coletados no município de Altos, Estado do Piauí, à latitude de $05^{\circ} 02^{\prime} 20^{\prime \prime} \mathrm{S}$, longitude de $42^{\circ} 27^{\prime} 39^{\prime \prime} \mathrm{O}$ e a $187 \mathrm{~m}$ de altitude, a $42 \mathrm{~km}$ ao nordeste de Teresina. O solo local é Podzólico Vermelho Amarelo Distrófico com baixa fertilidade natural (P $5 \mathrm{mg} \cdot \mathrm{dm}^{-3}, \mathrm{~K} 14 \mathrm{mg} \cdot \mathrm{dm}^{-3}$, Al 6 mmol.dm ${ }^{-3}$, e Ca+Mg 8 mmol.dm ${ }^{-3}$ ) e alta acidez (pH - 5,3). A precipitação média anual do município é em torno de $1.297 \mathrm{~mm}$, sendo que cerca de $90 \%$ das chuvas concentram-se no período de novembro a maio. A temperatura média anual está em torno de $25^{\circ} \mathrm{C}$ e a umidade relativa de $67 \%$. A identificação botânica foi realizada no Núcleo de Referência em Ciências Ambientais do Trópico Ecotonal do Nordeste - TROPEN, Teresina - PI, sendo a exsicata depositada sob o número 21.644 no herbário Graziela Barroso.

Os frutos foram picados, dessecados em estufa de circulação forçada de ar, durante 8 dias, a uma temperatura máxima de $45^{\circ} \mathrm{C}( \pm 1)$. Após essa etapa, o material foi triturado em moinho, tipo Willis, obtendo-se um pó que foi acondicionado em um frasco de vidro âmbar hermeticamente fechado e identificado, onde permaneceu até o momento do preparo dos extratos.

\section{Preparação dos extratos}

O extrato etanólico foi obtido através de maceração a frio, após quatro extraçóes sucessivas, sendo filtrado usando-se papel de filtro Whatman $\mathrm{n}^{\circ} 1$. Em seguida, concentrado em evaporador rotativo sob pressão reduzida, à temperatura entre 42 e $45^{\circ} \mathrm{C}$ e, entấo, liofilizado. Utilizou-se em média 1,3 L de etanol para cada extração realizada. $\mathrm{O}$ extrato aquoso foi obtido através de cocção, ou seja, $50 \mathrm{~g}$ de pó da matéria vegetal para $500 \mathrm{~mL}$ de água destilada e, em seguida, deixou-se ferver por dois minutos e, após o resfriamento da temperatura ambiente, a solução obtida foi filtrada, obtendo-se então uma solução a $10 \%$.

\section{Determinação do peso seco}

Foi retirada uma alíquota de $1 \mathrm{~mL}$ de cada extrato, colocada em estufa de circulação forçada de ar, a uma temperatura de $45^{\circ} \mathrm{C}( \pm 1)$, até a obtenção de um peso constante, procedimento realizado em triplicata. A massa média obtida referente a $1 \mathrm{~mL}$ foi relacionada ao respectivo volume total, obtendo-se entâo a massa total em mg. $\mathrm{mL}^{-1}$. Esse procedimento foi realizado para o extrato aquoso com o objetivo de determinar o peso seco e, para o extrato etanólico, para avaliar o rendimento aproximado após a evaporaçấo do etanol. 


\section{Manutenção das aves}

Durante o experimento, foram adquiridas em granjas da zona rural, da cidade de Teresina - PI, galinhas poedeiras em fase de descarte e que não recebiam vermífugo há pelo menos três meses. Foram colocadas em um galpão composto por uma área coberta, com bebedouros e comedouros, além da cama de maravalha. Os animais tiveram acesso a uma área descoberta e de chão batido, capinada, evitando-se que os animais tivessem acesso a outras plantas. Para determinar o grau de infecção do plantel, foram realizados exames de fezes, pela técnica Willis modificada (WILLIS apud UENO, 1994).

\section{Atividade anti-helmintica "in vitro"}

Foi determinada em helmintos adultos, machos e fêmeas de $A$. galli, coletados a partir do intestino delgado de aves necropsiadas. Os parasitos foram lavados em soluçáo salina $0,9 \%$, e aqueles considerados ativos foram transferidos para placas de Petri descartáveis $(150 \times 15 \mathrm{~mm})$, contendo solução Tyrode (KALEYSA, 1975) pré-aquecida $\left(37^{\circ} \mathrm{C}\right)$, totalizando 10 nematoides por placa. A seguir, foram adicionados os extratos nas seguintes concentraçôes: 1,69; 3,37; 6,74; 13,48 e 26,96 mg. $\mathrm{mL}^{-1}$ do extrato aquoso; e 4,17; 8,34; 16,68; 33,36 e $66,72 \mathrm{mg} \cdot \mathrm{mL}^{-1}$ do extrato etanólico. Os nematoides foram mantidos em estufa BOD, à temperatura de $37^{\circ} \mathrm{C}( \pm 1)$, e examinados $6,24,48$, 72 e 96 horas após o tratamento. Os nematoides imóveis, mesmo após breve pressão com estilete, foram considerados mortos (SHILASKAR; PARASAR, 1985). O experimento foi repetido 3 vezes para cada dose, e a porcentagem de parasitas mortos em cada grupo foi calculada. Esse teste foi acompanhado de dois controles negativos constituídos por água e diluente (Tween 80 a 17,5\% acrescido de DMSO a $12,5 \%$ ) e um controle com a droga referência, citrato de piperazina $\left(50 \mathrm{mg} \cdot \mathrm{mL}^{-1}\right)$, administrada de acordo com o fabricante (Proverm Tortuga).

\section{Atividade anti-helmintica "in vivo"}

Os grupos $(\mathrm{n}=6)$ compostos de aves com peso médio de $1,5 \mathrm{~kg}$, foram constituídos por controle positivo (piperazina, $50 \mathrm{mg} \cdot \mathrm{mL}^{-1}$ ), controles negativos (água e diluente idêntico ao teste in vitro) e grupos tratados com os extratos aquoso e etanólico de $M$. citrifolia, nas doses de 50,1 e 24,6 mg. $\mathrm{mL}^{-1}$, respectivamente. Diagnosticada a infecção por exame de fezes, as aves foram transferidas para gaiolas individuais galvanizadas, com fundo removível, onde passaram por um período de adaptaçáo de 72 horas, recebendo diariamente $50 \mathrm{~g}$ de ração e água ad libitum. Antes do início dos testes, as aves foram submetidas a um período de jejum de 6 horas, tendo disponível água à vontade. Os extratos aquoso e etanólico foram administrados, durante três dias consecutivos, no volume de $10 \mathrm{~mL} \cdot \mathrm{kg}^{-1}$, utilizando-se uma sonda intragástrica (FERNANDES et al., 2005). As fezes foram coletadas durante quatro dias por grupo. Em seguida, foram levadas ao laboratório, lavadas em água corrente e peneiradas em tamis USBS - 50, abertura $0,297 \mathrm{~mm}$ e tyler 48 sob outro tamis de malha USBS - 40, abertura $0,42 \mathrm{~mm}$ e tyler 35 colocado no fundo da pia, de modo a reter o resíduo que passasse pelo primeiro tamis. Posteriormente, foram acondicionadas em frascos contendo uma solução AFA a quente (Ácido Acético, Formol e Álcool) (AMATO et al., 1991) para conservação, visando à contagem dos helmintos eliminados. No quinto dia de tratamento, as aves foram sacrificadas e necropsiadas de acordo com a Resolução no 714 de 20/06/2002 do Conselho Federal de Medicina Veterinária. A mucosa do trato gastrintestinal foi raspada e o conteúdo colocado em frascos contendo AFA quente para posterior contagem e identificação dos helmintos remanescentes. O efeito do tratamento foi avaliado pelo método crítico controlado (STEWARD, 1955), adaptado ao presente modelo experimental, e expresso em percentual médio de eliminação de A. galli, conforme a Equaçáo 1 abaixo:

$\begin{aligned} & \text { Ante atividade } \\ & \text { Antimintica }\end{aligned}=\frac{\begin{array}{c}\text { No total de nematoides eliminados } \\ \text { nas fezes após o tratamento }\end{array}}{\begin{array}{c}\text { No total de nematoides } \\ \text { eliminados nas fezes } \\ \text { após o tratamento }\end{array}+\begin{array}{c}\text { No total de nematoides } \\ \text { recuperados na necropsia }\end{array}} \times 100(1)$

Cálculo semelhante foi aplicado aos grupos controles negativos.

\section{Análise estatística}

Os dados obtidos foram analisados estatisticamente, utilizando-se o teste Student-Newman-Keuls, com auxílio do programa InStat (Graphpad Instat: GraphPad Software Oberlin, San Diego - CA, USA). O nível de significância adotado foi $\mathrm{P}<0,05$ (PIMENTEL-GOMES, 1987).

\section{Resultados e Discussão}

Morinda citrifolia apresentou, no extrato etanólico, rendimento elevado, no qual se obteve a quantidade de $125 \mathrm{~g}$ do extrato na forma de pó depois do processo de liofilização. Esse resultado foi expressivo, pois, segundo Rates (2001), a grande dificuldade na realização de ensaios clínicos está relacionada com a quantidade de extrato e de princípio ativo obtido das plantas. $\mathrm{O}$ pH do extrato etanólico do fruto do noni foi de 4,40 e, do extrato aquoso, de 3,86 . O peso seco do extrato aquoso do fruto do noni foi de $50,1 \mathrm{mg} \cdot \mathrm{mL}^{-1}$ e, do extrato etanólico, de $24,6 \mathrm{mg} \cdot \mathrm{mL}^{-1}$.

A percentagem média de mortalidade nos testes in vitro, usando-se diferentes concentraçôes do extrato aquoso do fruto de $M$. citrifolia, em diferentes tempos de exposição do parasito ao extrato, está disposta na Tabela 1 .

$\mathrm{O}$ extrato aquoso do noni começou a apresentar efeito a partir de 48 horas na concentraçáo de $26,96 \mathrm{mg} \cdot \mathrm{mL}^{-1}$, sendo esse efeito não significativo estatisticamente, quando comparado com o controle negativo $(P>0,05)$. Porém, na $72^{\circ}$ e $96^{\circ}$ hora nas concentraçôes de 13,48 e $26,96 \mathrm{mg} \cdot \mathrm{mL}^{-1}$, houve diferença estatisticamente significativa, considerando-se o extrato aquoso do fruto do noni, o controle positivo (piperazina) e o controle negativo (água). Comparando-se as taxas de mortalidade do tratamento e do controle negativo, observa-se que, nos dois últimos períodos de tempo, há uma maior discrepância da eficácia do extrato aquoso do fruto do noni com relação ao teste com água. 
Tabela 1. Percentual médio de mortalidade do extrato aquoso do fruto de Morinda citrifolia sobre Ascaridia galli, no teste in vitro, ao longo de 96 horas de tratamento.

\begin{tabular}{|c|c|c|c|c|c|c|}
\hline & \multirow{2}{*}{$\begin{array}{c}\text { Concentraçáo } \\
\left(\mathbf{m g} \cdot \mathbf{m L}^{-1}\right)\end{array}$} & \multicolumn{5}{|c|}{ Tempo (horas) / Taxa de Mortalidade (\%) } \\
\hline & & 6 & 24 & 48 & 72 & 96 \\
\hline \multirow{5}{*}{$\begin{array}{l}\text { Extrato aquoso } \\
\text { do noni }\end{array}$} & 1,69 & 0 & 0 & 0 & 0 & 0 \\
\hline & 3,37 & 0 & 0 & 0 & 0 & $6,67^{a}$ \\
\hline & 6,74 & 0 & 0 & 0 & $6,67^{a}$ & $40^{\mathrm{b}}$ \\
\hline & 13,48 & 0 & 0 & 0 & $16,67^{b}$ & $46,67^{\mathrm{b}}$ \\
\hline & 26,96 & 0 & 0 & $3,33^{a}$ & $16,67^{b}$ & $50^{\mathrm{b}}$ \\
\hline Piperazina & 50 & 0 & 0 & 0 & $50^{c}$ & $100^{c}$ \\
\hline Água & & 0 & 0 & $0,67^{a}$ & $5,35^{\mathrm{a}}$ & $14^{\mathrm{a}}$ \\
\hline
\end{tabular}

Médias com letras iguais, na coluna, náo diferem entre si pelo teste de Student-Newman-Keuls $(\mathrm{P}>0,05)$.

Tabela 2. Percentual médio de mortalidade do extrato etanólico do fruto de Morinda citrifolia sobre Ascaridia galli, no teste in vitro, ao longo de 96 horas de tratamento.

\begin{tabular}{|c|c|c|c|c|c|c|}
\hline & \multirow{2}{*}{$\begin{array}{l}\text { Concentraçáo } \\
\left(\mathbf{m g} \cdot \mathbf{m L}^{-1}\right)\end{array}$} & \multicolumn{5}{|c|}{ Tempo (horas) / Taxa de Mortalidade (\%) } \\
\hline & & 6 & 24 & 48 & 72 & 96 \\
\hline \multirow{5}{*}{$\begin{array}{l}\text { Extrato etanólico } \\
\text { do noni }\end{array}$} & 4,17 & 0 & 0 & 0 & $6,67^{a}$ & $23,33^{\mathrm{a}}$ \\
\hline & 8,34 & 0 & 0 & 0 & $3,33^{\mathrm{a}}$ & $26,67^{a}$ \\
\hline & 16,68 & 0 & 0 & 0 & $6,67^{a}$ & $33,33^{b}$ \\
\hline & 33,36 & 0 & 0 & $6,67^{a}$ & $33,33^{\mathrm{b}}$ & $66,67^{\mathrm{b}}$ \\
\hline & 66,72 & 0 & 0 & $10^{\mathrm{a}}$ & $33,33^{\mathrm{b}}$ & $76,67^{\mathrm{b}}$ \\
\hline Piperazina & 50 & 0 & 0 & 0 & $50^{c}$ & $100^{c}$ \\
\hline Diluente* & & 0 & 0,67 & $3,33^{\mathrm{a}}$ & $6,67^{a}$ & $13,33^{\mathrm{a}}$ \\
\hline
\end{tabular}

Médias com letras iguais, na coluna, não diferem entre si pelo teste de Student-Newman-Keuls (P > 0,05). *(Tween 80 a 17,5\% acrescido de DMSO a 12,5\%).

O extrato etanólico apresentou diferença estatisticamente significativa do controle negativo (diluente) na 96 hora nas concentraçóes de 33,36 e 66,72 mg. $\mathrm{mL}^{-1}(\mathrm{P}<0,05)$. A maior concentração do extrato etanólico do fruto do noni $\left(66,72 \mathrm{mg} \cdot \mathrm{mL}^{-1}\right)$ demonstrou melhor eficiência no combate ao parasito, sendo a taxa de mortalidade de 76,67\% (Tabela 2).

Quarenta e oito horas pós-tratamento, foi verificado que tanto o extrato aquoso como o etanólico do fruto do noni, demonstraram efeito anti-helmíntico superior ao da piperazina na concentração mais elevada. Considerando-se a mortalidade acumulada ao longo das 96 horas, verifica-se que o extrato etanólico apresentou um maior percentual de mortalidade do que o extrato aquoso. Hadinoto e Hadisoewignyo (2003) avaliando o efeito anti-helmíntico do suco do noni sobre o $A$. galli em várias concentrações $(7,9,12$, 16 , e $21 \%$ ), observaram atividade anti-helmíntica satisfatória no teste in vitro, sendo também superior à da piperazina utilizada em diferentes concentraçóes (20, 25, 32, 40 e 50\%).

Em experimentos semelhantes, o suco de noni foi testado in vitro em várias concentraçóes sobre Ascaris suum, obtendo-se mortalidade de $100 \%$ na concentração de $60 \%$. Porém a solução de piperazina a $20 \%$ demonstrou taxa de mortalidade de $60 \%$, inferior ao que se obteve na mesma concentração com o suco do noni (KESEHATAN, 2003), resultado que diferiu do encontrado neste trabalho, provavelmente devido à diferentes níveis de sensibilidade dos parasitos ao anti-helmíntico. Murdiati et al. (2000) pesquisaram a atividade anti-helmíntica do noni em caprinos e ovinos, levando em consideração Haemonchus contortus e afirmaram que, no teste in vitro, o extrato clorofórmico foi eficiente na morte de parasitos adultos e no desenvolvimento dos ovos, obtendo melhores resultados do que os encontrados nesta pesquisa.

De acordo com Heinicke (1985), a fruta noni contém um precursor natural da xeronina, a proxeronina. Esta substância após ingestão é convertida no alcalóide xeronina através da enzima proxeroninase. Heinicke (1985), afirma que a xeronina é capaz de modificar a estrutura molecular das proteínas. Desse modo, a xeronina tem uma ampla escala de atividades biológicas. Quando uma proteína, tal como uma enzima, receptora ou transdutora de sinal não está em sua conformação apropriada, ela não desempenhará sua função corretamente. Então, a xeronina irá interagir com a proteína e fazer com que ela retorne à conformação adequada. O resultado é o funcionamento correto da atividade da proteína. No caso de haver um problema estrutural da proteína, a presença da xeronina será benéfica.

Os dados da Tabela 3 mostram, no teste in vivo, os percentuais de eliminação de $A$. galli em aves poedeiras naturalmente infectadas, submetidas à administração de extrato aquoso e etanólico de M. citrifolia. Comparando-se estatisticamente os três tratamentos, verifica-se que houve diferença significativa entre o extrato aquoso, controle negativo (água) e controle padráo (piperazina) $(\mathrm{P}<0,05)$, demonstrando $27 \%$ de eficiência do fruto de $M$. citrifolia na eliminação do $A$. galli. No entanto, de acordo com a classificação do índice de eficácia proposto pela World Association for the Advancement of Veterinary Parasitology (W.A.A.V.P.) (POWERS et al., 1982), um produto seria altamente efetivo se apresentasse mais de 
Tabela 3. Atividade anti-helmíntica dos extratos aquoso e etanólico obtidos do fruto de Morinda citrifolia na eliminação de Ascaridia galli em aves poedeiras $(\mathrm{n}=6)$ naturalmente infectadas.

\begin{tabular}{lccc}
\hline \multirow{2}{*}{ Tratamentos } & \multicolumn{2}{c}{ Número de helmintos } & \multirow{2}{*}{ Eliminaçáo $(\%)$} \\
\cline { 2 - 3 } & Exame de fezes & Necropsia & $27,08^{\mathrm{a}}$ \\
Extrato aquoso $\left(50,1 \mathrm{mg} \cdot \mathrm{mL}^{-1}\right)$ & 26 & 70 & $8,11^{\mathrm{b}}$ \\
Extrato etanólico $\left(24,6 \mathrm{mg} \cdot \mathrm{mL}^{-1}\right)$ & 06 & 68 & $1,96^{\mathrm{b}}$ \\
Água & 01 & 50 & $3,64^{\mathrm{b}}$ \\
Diluente* & 02 & 53 & $98,61^{\mathrm{c}}$ \\
Piperazina $\left(50 \mathrm{mg} \cdot \mathrm{mL}^{-1}\right)$ & 498 & 07 & \\
\hline
\end{tabular}

Médias com letras iguais, na coluna, não diferem entre si pelo teste de Student-Newman-Keuls (P > 0,05). *(Tween 80 a 17,5\% acrescido de DMSO a 12,5\%).

90\% de ação contra o parasito tratado; moderadamente efetivo quando atuasse entre 80 a $90 \%$; pouco efetivo quando a ação fosse entre 60 e $80 \%$; e não efetivo em níveis abaixo de $60 \%$. Sabe-se que o extrato de uma planta possui diversas substâncias, sendo uma delas o princípio ativo, enquanto produtos industrializados possuem esse princípio isolado, apresentando, assim, uma eficácia mais elevada. Portanto, o extrato aquoso do fruto do noni na concentraçáo de $10 \%$, não foi efetivo na eliminação de $A$. galli em aves poedeiras infectadas naturalmente. Verifica-se que o extrato etanólico da $M$. citrifolia não apresentou diferença estatística significativa em relação ao controle (diluente) $(\mathrm{P}>0,05)$, havendo apenas diferença estatística em relação ao controle positivo, demonstrando não ser efetivo na eliminação de $A$. galli.

Os resultados obtidos no teste in vivo, considerando-se a concentração de 10\%, demonstraram uma baixa eficiência da ação do extrato do fruto (aquoso e etanólico) frente a $A$. galli, não descartando a possibilidade de extratos com concentraçóes mais elevadas apresentarem atividade anti-helmíntica efetiva. No teste in vitro, os extratos demonstraram ser pouco efetivos sobre a taxa de mortalidade. Portanto, faz-se necessária a continuidade da pesquisa com o objetivo de obter mais informaçóes sobre a atividade parasiticida de $M$. citrifolia.

\section{Referências}

AMATO, J. F. R.; BOEGER, W. A.; AMATO, S. B. Protocolos para laboratório: coleta e processamento de parasitos de pescado. Rio de Janeiro: Imprensa Universitária, 1991.77 p.

CAMURÇA-VASCONCELOS, A. L. F. et al. Validação de plantas medicinais com atividade anti-helmíntica. Revista Brasileira de Plantas Medicinais, v. 7, n. 3, p. 97-106, 2005.

FERNANDES, R. M. et al. Atividade anti-helmíntica de plantas em frangos de corte naturalmente infectados com Ascaridia galli. Arquivo Brasileiro de Medicina Veterinária, v. 57, supl. 2, p. 264-266, 2005.

FREITAS, M. G. Helmintologia Veterinária. Belo Horizonte: Rabelo \& Brasil, 1977. p. 397.

HADINOTO, I.; HADISOEWIGNYO, D. L. Potential anthelmintic effect of the juice of Morinda citrifolia Linn. on Ascaridia galli in vitro. Media Kedokteran Hewan, v. 20, n. 2, p. 19-22, 2003.

HEINICKE, R. M. The pharmacologically active ingredient of noni. Pacific Tropical Botanical Garden Bulletin, v. 15, n. 1, p. 10-14, 1985.
KALEYSA, R. R. Screening of indigenous plants for anthelmintic action against human Ascaris lumbricoides. Indian Journal Physiology Pharmacology, v. 19, n. 1, p. 47-49, 1975. (part II)

KESEHATAN, B. L. Efek Antelmintik Mengkudu (Morinda citrifolia L.) terhadap Ascaris suum in vitro/Ludmilla. Jkpkbppk: Jakarta, Indonésia, 2003. Disponível em: <http://www.jkpkbppk-gdl-res-2003-budikusuma1687-anthelmint>. Acesso em: 08 de Janeiro de 2008.

MARTINS, E. R. et al. Plantas medicinais. Viçosa: UFV, 2000. 220 p.

MURDIATI, T. B.; ADIWINATA, G.; HILDASARI, D. To trace the active compound in mengkudu (Morinda citrifolia) with anthelmintic activity against Haemonchus contortus. Jurnal Ilmu Ternak dan Veteriner, v. 5, n. 4, p. 255-259, 2000.

PERMIN, A. et al. Ecto-, Endo- and haemoparasites in free-range chickens in the Goromonzi District in Zimbabwe. Preventive Veterinary Medicine, v. 54, n. 3, p. 213-224, 2002.

PIMENTEL-GOMES, F. Curso de estatística experimental. 12 ed. Piracicaba: Nobel, 1987. 467 p.

POWERS, K. G. et al. World Association for the Advancement of Veterinary Parasitology (W.A.A.V.P.): guidelines for evaluating the efficacy of anthelmintics in ruminants (bovine and ovine). Veterinary Parasitology, v. 10, n. 4 , p. 265-284, 1982.

RATES, S. M. K. Plants as source of drugs. Toxicon, v. 39, n. 5, p. 603-613, 2001.

SHILASKAR, D. V.; PARASAR, G. C. In vivo and kymographic studies on Psoralea corylifolia and piper betle against avian Ascaridia galli. Indian Veterinary Journal, v. 62, n. 5, p. 387-394, 1985.

STEWARD, J. S. Anthelmintic studies: I a controlled critical enteronemacidal test. Parasitology, v. 45, n. 3-4, p. 231-241, 1955.

THAMSBORG, S. M.; ROEPSTORFF, A.; LARSEN, M. Integrated and biological control of parasites in organic and conventional production systems. Veterinary Parasitology, v. 84, n. 3-4, p. 169-186, 1999.

VIEIRA, L. S. et al. Evaluation of anthelmintic efficacy of plants available in Ceará State, North - East Brazil, for the control of goat gastrointestinal nematodes. Revue de Medicine Vétérinaire, v. 150, n. 5, p. 447-452, 1999.

WANG, M. Y. et al. Morinda citrifolia (Noni): a literature review and recent advances in noni research. Acta Pharmacologica Sinica, v. 23, n. 12, p. 1127-1141, 2002.

WILLIS, H. H. A simple levitation method for the detection of hookworm ova. In: UENO, H.; GONÇALVES, P. C. Manual para dignóstico das helmintoses de ruminantes. 3 ed. Tokyo: Japan International Cooperation Agency, 1994. p. 14.

ZHAN, J.; ZHOU, P. A. A simplified method to evaluate the acute toxicity of ricin and ricius agglutinin. Toxicology, v. 186, n. 1-2, p. 119-123, 2003. 